\title{
Telemonitoring in heart failure: fact, fiction, and controversy
}

\author{
This article was published in the following Dove Press journal: \\ Smart Homecare Technology and TeleHealth \\ 14 October 2015 \\ Number of times this article has been viewed
}

\author{
Sally C Inglis \\ Centre for Cardiovascular and \\ Chronic Care, Faculty of Health, \\ University of Technology Sydney, \\ Australia
}

Correspondence: Sally C Inglis

Centre for Cardiovascular and Chronic

Care, Faculty of Health, University of Technology Sydney, PO Box 123 , Broadway, NSW 2007, Australia

Tel +61295144819

Fax +6I 295 I 44474

Email sally.inglis@uts.edu.au
Abstract: The facts, fiction, and controversial issues regarding contemporary use of telemonitoring in heart failure are discussed, along with implications for future research and clinical practice. Recent studies labeled as telemonitoring have reported findings inconsistent with large systematic reviews and meta-analyses of the literature on telemonitoring in heart failure. This review explores some of the reasons why these inconsistencies may exist and also discusses some of the key issues in the contemporary evidence and use of telemonitoring in heart failure.

Keywords: heart failure, telemonitoring, remote monitoring, mortality, hospitalization

\section{Introduction}

Cardiovascular disease remains the leading cause of death globally, with heart failure a main contributor to these mortality statistics. ${ }^{1}$ Heart failure is a prevalent, complex, and burdensome syndrome that will continue to be responsible for significant health care cost into the future. ${ }^{2}$ Despite improvements in the treatment of cardiovascular disease, it is predicted that longer survival, combined with an aging population and increasing rates of obesity, will see rises in health care costs as well as disability, and in turn, negative impacts on quality of life. ${ }^{3}$ The current and future burden of cardiovascular disease will require innovative and cost-effective strategies to manage this burden.

The demand placed on the health care system through frequent hospitalizations for people with heart failure and the potential to improve patient outcomes through education, self-management, monitoring, and specialized care ${ }^{4}$ has led to heart failure management programs that are recommended as "gold-standard" heart failure care internationally. ${ }^{5,6}$ Home monitoring is an essential element of heart failure management, ${ }^{7}$ and involves both the patient and health care provider being alerted to any changes in signs and symptoms of heart failure that may indicate clinical worsening. Home monitoring of signs and symptoms can occur via a number of methods and for patients with heart failure typically involves monitoring of weight as well as symptoms, such as shortness of breath, and signs such as edema, and in some instances, blood pressure, heart rate, electrocardiograms, and medication adherence.

Telemonitoring (electronic transfer of physiological data, including electrocardiography, blood pressure, weight, pulse oximetry, and respiratory rate, as well as medication adherence and in some cases, symptoms) is a way in which people with heart failure can be monitored remotely by their clinicians, synchronously or asynchronously. For the purposes of this review, telemonitoring refers to the synchronous or asynchronous transfer of physiological and/or signs and symptom data using noninvasive monitoring 
devices. Despite the first trials of telemonitoring interventions in heart failure being conducted more than a decade ago, ${ }^{8,9}$ there is still controversy and debate regarding the effectiveness, suitability, and cost of telemonitoring for people with heart failure. ${ }^{10-12}$ This review examines the facts, fiction, and controversies regarding the use of telemonitoring in the management of heart failure. It focuses on the research evidence related to the application of telemonitoring rather than the ethical, legal, and cost aspects of telemonitoring, which fall outside the scope of this review.

There is a need to improve access to "gold standard" heart failure management programs in many parts of the world, ${ }^{13-17}$ and many patients with heart failure in the developed and developing world do not have access to regular specialized monitoring and support. Telemonitoring, along with other forms of remote monitoring or management such as structured telephone support may be ways of improving access to specialized monitoring and support; however, we have been hesitant to integrate telemonitoring into the care of people with heart failure. Why so? Despite the use of information communication technology in many other parts of our lives and greater rationalization of health care budgets, which may limit widespread provision of face-to face-monitoring services such as heart failure home nurses or clinics, telemonitoring is not accepted as "standard" care across the world, and current heart failure guidelines do not support remote management for patients with heart failure, ${ }^{5,6}$ due to inconsistent study findings and quality. There are key factors that may contribute to these issues and some are explored in this review.

\section{What is fact and what is fiction when it comes to telemonitoring in heart failure?}

Firstly, to get our facts straight, we need to be clear about what we are talking about when we use the term "telemonitoring". Patients can be remotely monitored and supported using an ever increasing number of information communication technologies and devices. In the current heart failure literature, these include structured telephone or videophone support (a person-to-person conversation following a structured format between a patient and typically a health professional such as a nurse), an interactive voice response system, and synchronous or asynchronous transfer of physiological, hemodynamic, and/or symptom data using invasive and/or noninvasive monitoring devices. In this review, telemonitoring refers to the synchronous or asynchronous transfer of physiological and/or signs and symptom data using noninvasive monitoring devices. Many studies of remote monitoring interventions are referred to as "telemonitoring", but the intervention may actually be structured telephone support or an interactive voice response system. The inconsistent use of these terms has probably to some extent contributed to the heterogeneity observed and critiqued in the findings between different studies of "telemonitoring" and in turn the effectiveness that may or may not be credited to telemonitoring in heart failure.

There is a significant need for standardization in reporting and defining of interventions that involve any form of remote monitoring so as to reduce inconsistencies in use of the many different terms, and thereby allow appropriate comparison across studies of similar interventions in order to evaluate the effectiveness of remote monitoring accurately and transparently.

There are several reviews of telemonitoring in heart failure, ${ }^{18-25}$ all with varying methodologies (ie, including both randomized and nonrandomized studies), study inclusion criteria (ie, including classification of what constitutes telemonitoring versus other forms of remote monitoring), and quality. ${ }^{26,27}$

Evidence-based health care places value on good quality systematic reviews of randomized controlled trials to inform practice. ${ }^{28}$ Although systematic reviews are considered the highest level of evidence, it is important to remember that heterogeneity often exists across studies included in a systematic review in terms of populations, interventions, and outcomes (clinical diversity), as well as in study design and risk of bias (methodological diversity). ${ }^{1}$

A Cochrane review published in 2010 included eleven peer-reviewed, published trials of telemonitoring, involving 2,710 participants. ${ }^{18}$ Separate meta-analyses and comparisons were performed for structured telephone support and are not discussed here. Telemonitoring was shown to reduce all-cause mortality (relative risk [RR] 0.66, 95\% confidence interval [CI] 0.54-0.81, $P<0.0001)$, heart failure-related hospitalizations (RR $0.79,95 \%$ CI $0.67-0.94, P=0.008$ ), and the risk of all-cause hospitalizations (RR 0.91, 95\% CI $0.84-0.99, P=0.022) .{ }^{18}$ A recent overview of systematic reviews of telemonitoring in cardiovascular disease identified this Cochrane review ${ }^{18}$ to be of good quality and other more recent reviews of telemonitoring in heart failure to have more concerns with their methodology. ${ }^{26}$

Since publication of the Cochrane review in 2010, ${ }^{18}$ additional studies of remote monitoring in heart failure have been published, ${ }^{29-33}$ and have reported mixed findings regarding the effectiveness of these interventions 
with regard to outcomes. These recent findings have raised questions about the effectiveness and suitability of telemonitoring and other forms of remote management in heart failure. Not all of the recent studies of remote monitoring in heart failure can be classed as telemonitoring, so labeling as such has probably led to some misinterpretation of the current evidence for the effectiveness of telemonitoring in heart failure. Some of the recent studies of remote monitoring that have been discussed in relation to their findings and current evidence for the effectiveness of telemonitoring are highlighted here (see Table 1).

TIM-HF (the Telemedical Interventional Monitoring in Heart Failure Study) ${ }^{29}$ reported a neutral effect of telemonitoring in 710 patients $(>80 \%$ male) with stable heart failure, who had a mean age of 66.9 years, were in New York Heart Association functional class II or III, and had a left ventricular ejection fraction of $\leq 35 \% .{ }^{29}$ The telemonitoring intervention consisted of electronic transfer of a three-lead electrocardiogram, blood pressure, and weight daily, with physician-led medical support available 24 hours, 7 days a week, that also included the capacity to initiate treatment in response to data transferred. No effect of telemonitoring was observed with regard to all-cause mortality (hazard ratio $0.97 ; 95 \%$ CI $0.67-1.41, P=0.87$ ) or on cardiovascular death or heart failure hospitalization (hazard ratio $0.89 ; 95 \% \mathrm{CI}$ $0.67-1.19, P=0.44){ }^{29}$

The TEHAF (Telemonitoring in patients with Heart Failure) study ${ }^{31}$ reported mainly neutral findings of a remote monitoring intervention in 382 patients (59\% male) of mean age 71.5 years, with the majority in New York Heart Association functional class II-IV. The median ejection fraction was $36 \% .{ }^{31}$ Unlike other telemonitoring studies, this study utilized a device that asked the patient about their symptoms and health behaviors and communicated this to a heart failure nurse rather than automatically transmitting vital signs. Despite communicating different information when compared with the TIM-HF study, the TEHAF study did not observe a benefit in terms of all-cause mortality (9.1\% for the intervention group versus $6.5 \%$ for the control group; $P=0.34$ ) or the combined endpoint of heart failure hospitalization and all-cause mortality (hazard ratio 0.89 ; 95\% CI 0.69-1.83, $P=0.641){ }^{31}$

The WISH (Weight monitoring In patients With Severe Heart failure) study $^{30}$ reported neutral findings as well. ${ }^{30}$ Conducted in Sweden, WISH examined the benefit of daily electronic transmission of body weight in 319 patients hospitalized with New York Heart Association functional class III to IV heart failure, $57 \%$ of whom had a left ventricular ejection fraction of $<30 \% .^{30}$ The mean age of the study participants was 73 years and $75 \%$ were male. No statistically significant difference was found for the primary end point of cardiac rehospitalization (hazard ratio $0.90 ; 95 \%$ CI $0.65-1.26, P=0.54)$, the secondary endpoint of all-cause mortality (hazard ratio $0.57,95 \%$ CI $0.19-1.73, P=0.32$ ), or the combined endpoint of cardiac hospitalization and all-cause mortality (hazard ratio 0.90; 95\% CI 0.65-1.26, $P=0.54) .^{30}$

The TEMA-HF 1 (TElemonitoring in the MAnagement of Heart Failure) study ${ }^{32}$ reported positive benefits of telemonitoring in 160 patients ( $65 \%$ male) with a mean age of 77 years and a median left ventricular ejection fraction of $35 \% .{ }^{32}$ Participants in the telemonitoring study arm were asked to measure their weight, blood pressure, and heart rate each morning at a set time. Blood pressure and weight measurements were automatically forwarded, with alerts sent to the patients' general practitioners and to the heart failure clinic if the measurements were outside preset parameters for two consecutive days. This study reported a significant benefit of telemonitoring on all-cause mortality ( $5 \%$ versus $17.5 \%$, $P=0.01$ ) as well as total days lost because of hospitalization, dialysis, or death (13 versus 30 days, $P=0.02){ }^{32}$

Tele-HF (Telemonitoring to Improve Heart Failure Outcomes) $)^{33}$ is another study of remote monitoring which is often discussed in the context of studies that raise questions regarding the effectiveness of telemonitoring because of its neutral findings. According to the definition of telemonitoring applied in this current review, the intervention trialed in the Tele-HF study would be considered an interactive voice response system. ${ }^{34}$

The variation in these selected recent study finding $s^{29-33}$ considered in light of the findings of the large Cochrane review ${ }^{18}$ has raised several important questions about the patient population that can most benefit from telemonitoring and remote monitoring. ${ }^{35}$ In particular, what parameters should be measured, at what intensity, and are there any incremental gains from monitoring patients who are already well managed. ${ }^{36}$ These are important questions for future research, and studies that are currently underway will hopefully provide more clarity to the debate in conjunction with an update of the Cochrane review of telemonitoring and structured telephone support in chronic heart failure.

Ever since telemonitoring has been evaluated for monitoring and supporting patients with heart failure, anecdotally some individuals argue that patients with heart failure are typically elderly and could not or would not want to use telemonitoring. The reasons considered often concern 
Table I Selected recent studies of telemonitoring in heart failure

\begin{tabular}{|c|c|c|c|}
\hline Study & Participants and country & Intervention, comparison, and follow-up & Outcomes \\
\hline TIM-HF²9 & $\begin{array}{l}710 \text { stable chronic HF patients } \\
\text { Mean age } 66.9 \text { years } \\
81.3 \% \text { male } \\
\text { Eligibility criteria: NYHA Class II } \\
\text { or III with LVEF } \leq 35 \% \text { and } \\
\text { HF decompensation in last } \\
2 \text { years or LVEF } \leq 20 \% \\
\text { Germany }\end{array}$ & $\begin{array}{l}\text { Intervention: electronic transfer of three-lead } \\
\text { ECG, blood pressure, and weight daily. } 24 \text { hours } \\
\text { per day, } 7 \text { days per week, physician-led medical } \\
\text { support with capacity to initiate treatment } \\
\text { response to transferred data. } \\
\text { Comparison: usual care. } \\
\text { Median follow-up was } 26 \text { months. }\end{array}$ & $\begin{array}{l}\text { Primary end point was all-cause mortality. } \\
\text { All-cause mortality (HR 0.97; } \\
95 \% \mathrm{Cl} 0.67-\mathrm{I} .4 \mathrm{I} ; \mathrm{P}=0.87 \text { ). } \\
\text { Cardiovascular death or HF } \\
\text { hospitalization (HR } 0.89 \\
95 \% \mathrm{Cl} 0.67-1.19 ; P=0.44)\end{array}$ \\
\hline TEHAF $^{3 !}$ & $\begin{array}{l}382 \mathrm{HF} \text { patients } \\
\text { Mean age } 7 \mathrm{II} 5 \text { years } \\
59 \% \text { male } \\
\text { Eligibility criteria: NYHA Class II-IV, } \\
\text { "... at least one episode of } \\
\text { fluid retention requiring diuretics, } \\
\text { either with an echocardiographic } \\
\text { left ventricular ejection } \\
\text { fraction } \leq 40 \% \text { or a preserved } \\
\text { ejection fraction with diastolic } \\
\text { dysfunction ...." }{ }^{\prime} \\
\text { The Netherlands }\end{array}$ & $\begin{array}{l}\text { Intervention: health Buddy device electronically } \\
\text { communicated patient responses regarding } \\
\text { symptoms and health behaviors to heart } \\
\text { failure nurse. } \\
\text { Comparison: nurse-led usual care including } \\
\text { oral and written educational information, and } \\
\text { psychosocial support as needed. } \\
\text { I } 2 \text { months of follow-up. }\end{array}$ & $\begin{array}{l}\text { Primary end point was time to first heart } \\
\text { failure hospitalization. } \\
\text { Mean time to first HF-related } \\
\text { hospitalization was I } 6 \text { I days for the } \\
\text { intervention group and I } 39 \text { days for the } \\
\text { comparison; hospitalizations occurred } \\
\text { in I8 }(9.1 \%) \text { compared with } 25 \text { ( } 13.5 \%) \\
\text { patients (HR } 0.65,95 \% \mathrm{Cl} 0.35-\mathrm{I} .17 \text {; } \\
P=0.15 \mathrm{I}) \text {. } \\
\text { No differences were found regarding } \\
\text { secondary end points, except for } \\
\text { the reduced number of face to face } \\
\text { contacts with the heart failure nurse } \\
(P<0.00 \mathrm{I}) \text {. Mortality was } 18(9.1 \%) \text { in the } \\
\text { intervention group and I2 }(6.5 \%) \text { in the } \\
\text { usual-care group }(P>0.05) \text {. }\end{array}$ \\
\hline $\mathrm{WISH}^{30}$ & $\begin{array}{l}319 \mathrm{HF} \text { patients following } \\
\text { hospitalization } \\
\text { Mean age } 73 \text { years } \\
75 \% \text { male } \\
\text { Eligibility criteria: hospitalized } \\
\text { for HF with NYHA class III-IV } \\
\text { and treatment with diuretics } \\
\text { and at least one other drug } \\
\text { used in HF (ACE inhibitor, ARB, } \\
\text { beta-blocker, an aldosterone } \\
\text { antagonist, or digoxin) an } \\
\text { impaired LVEF }<50 \% \text {. } \\
\text { Sweden }\end{array}$ & $\begin{array}{l}\text { Participants in both groups recommended to } \\
\text { weigh themselves daily and contact the HF clinic } \\
\text { if sudden weight gain }>2 \mathrm{~kg} \text { in } 3 \text { days. Weights } \\
\text { of both the comparison and intervention groups } \\
\text { were checked by the HF nurses on Mondays, } \\
\text { Wednesdays, and Fridays. } \\
\text { Intervention: participants provided with an } \\
\text { electronic scale, weight automatically } \\
\text { transmitted to and monitored at the HF clinic. } \\
\text { Comparison: participants recommended to } \\
\text { weigh themselves daily and contact the HF } \\
\text { clinic if sudden weight gain }>2 \mathrm{~kg} \text { in } 3 \text { days. } \\
12 \text { months of follow-up. }\end{array}$ & $\begin{array}{l}\text { Primary end point was cardiac } \\
\text { rehospitalization. } \\
\text { No significant difference was found for } \\
\text { cardiac rehospitalization (HR } 0.90,95 \% \\
\mathrm{Cl} 0.65-1.26, P=0.54 \text { ). } \\
\text { No significant difference was found } \\
\text { for the secondary end points; all- } \\
\text { cause hospitalization (HR } 0.83,95 \% \mathrm{Cl} \\
0.6 \mathrm{I}-\mathrm{I} .13, P=0.24) \text {, death from any } \\
\text { cause }(\mathrm{HR} 0.57,95 \% \mathrm{Cl} 0.19-1.73 \text {, } \\
P=0.32 \text { ), or the composite end point } \\
\text { of cardiac hospitalization and death } \\
\text { from any cause (HR } 0.90,95 \% \mathrm{Cl} \\
0.65-1.26, P=0.54) \text {. }\end{array}$ \\
\hline TEMA-HF I ${ }^{32}$ & $\begin{array}{l}\text { I60 CHF patients } \\
\text { Mean age } 77 \text { years } \\
65 \% \text { male } \\
\text { Eligibility criteria: hospitalized } \\
\text { for fluid overload due to HF } \\
\text { requiring an increase or initiation } \\
\text { of diuretics and receiving ACE } \\
\text { inhibitor or ARB and with a } \\
\text { beta-blocker unless } \\
\text { contraindicated } \\
\text { Belgium }\end{array}$ & $\begin{array}{l}\text { Intervention: participants were asked to measure } \\
\text { weight, BP and heart rate daily, at a fixed time in } \\
\text { the morning. Data were automatically forwarded. } \\
\text { Prespecified alert limits were determined for } \\
\text { weight, systolic BP, and heart rate. When } \\
\text { recordings fell outside these limits for two } \\
\text { consecutive days, the GP and HF clinic were } \\
\text { alerted by email. The study protocol instructed } \\
\text { the GP to visit or contact the patient and to } \\
\text { adapt the treatment, if necessary. The HF nurse } \\
\text { contacted the patient by telephone I-3 days after } \\
\text { the alert to check if the intervention was effective. } \\
\text { Comparison: participants were followed up by } \\
\text { their GP who could refer the patients to their } \\
\text { cardiologist if needed. No intervention was } \\
\text { provided by the study nurse or the HF clinic team. } \\
6 \text { months of follow-up. }\end{array}$ & $\begin{array}{l}\text { Primary end point was all-cause mortality. } \\
\text { All-cause mortality was significantly lower } \\
\text { in the intervention group ( } 5 \% \text { versus } \\
17.5 \%, P=0.01 \text { ). } \\
\text { Secondary outcomes included a } \\
\text { composite end point of total number of } \\
\text { follow-up days lost to hospitalization, } \\
\text { dialysis, or death and was significantly } \\
\text { lower in the intervention group } \\
(13 \text { versus } 30 \text { days, } P=0.02 \text { ). }\end{array}$ \\
\hline
\end{tabular}

Abbreviations: $\mathrm{ACE}$, angiotensin-converting enzyme; $\mathrm{ARB}$, angiotensin II receptor antagonist; $\mathrm{BP}$, blood pressure; $\mathrm{CHF}$, congestive heart failure; $\mathrm{Cl}$, confidence interval; ECG, electrocardiogram; GP, general practitioner; HF, heart failure; HR, hazard ratio; LVEF, left ventricular ejection fraction; NYHA, New York Heart Association classification; TIM-HF, Telemedical Intervention Monitoring in Heart Failure study; TEHAF, Telemonitoring in Heart Failsure study; WISH, Weight Monitoring in patients with Severe Heart failure; TEMA-HF, Telemonitoring in the Management of Heart Failure study. 
cognitive problems, hearing or vision impairments, lack of experience with technology, and lack of ongoing support in the use of the technology. Studies have refuted these claims, providing evidence that typical elderly heart failure patients adapt well to using telemonitoring technologies, ${ }^{37}$ which are ultimately designed with the user in mind. Post hoc analyses of telemonitoring outcomes from the Cochrane review indicate no evidence of significant differences in the benefit of telemonitoring in heart failure according to age when studies were stratified according to the mean/median age of participants being $\geq 70$ years of age. ${ }^{38}$ These findings (all-cause mortality $\geq 70$ years of age, RR 0.56 ; 95\% CI $0.41-0.76$, $P=0.0002$ ) indicate that older patients with heart failure should not be restricted from access to telemonitoring. ${ }^{38}$ As the younger generations living in the current era of mHealth, eHealth, smartphones, tablets, and wireless devices for all aspects of our lives will become heart failure patients in the future, the question of whether older patients can use telemonitoring will become more and more redundant. However, like all management strategies, these need to be tailored to the individual patient and there may be some for whom telemonitoring is neither the ideal nor an appropriate intervention for personal or situational reasons.

Patient acceptance of telemonitoring interventions is vital to their success and will undoubtedly influence adherence to the monitoring protocol. Without the patient adhering to the recommended protocol for monitoring, it could be hypothesized that the benefits of telemonitoring interventions would be less than if performed at the optimum frequency and capacity. Three recent qualitative studies have examined patient acceptance and use of technology and telemonitoring in the management of heart failure ${ }^{39}$ and offer insight into this important issue.

Fairbrother et $\mathrm{al}^{40}$ conducted individual semistructured interviews with 18 patients (mean age 74 years, 61\% male) who were enrolled in a heart failure telemonitoring service in Scotland. ${ }^{40}$ Patients liked the telemonitoring, found it easy to use, liked the reassurance that it provided, and reported that their knowledge and understanding of the condition increased. Patients reported some concerns with the device, such as technical issues, intrusiveness of the equipment in terms of size and noise from the device, cabling, and the costs of the equipment, installation, and ongoing monitoring. Some patients also preferred that a health care professional with whom they had an existing relationship be the person monitoring and responding to their telemonitored data. ${ }^{40}$

Hall et $\mathrm{al}^{41}$ conducted individual semistructured interviews with 15 heart failure patients (mean age 64 years,
$66 \%$ male) to assess their current use of technology for selfmanagement of heart failure. ${ }^{41}$ The authors of this study reported that all study participants responded positively when asked about using technology to assist them to improve or manage their own health. Key themes that emerged from these interviews related to some participants having concerns about being overwhelmed by technology; the objectivity of the data that the technology collected and having this recorded automatically; and increased access to health care and facilitation of real-time communication and information with health care professionals. ${ }^{41}$

Patient acceptance of telemonitoring should continue to be assessed so that devices and programs can be further refined to improve design of units and their suitability for patients as well as technical aspects. Clinician acceptance is also an important aspect in implementation of telemonitoring, particularly in a clinical rather than a research setting. Fairbrother et $\mathrm{al}^{40}$ canvassed the views of five professional staff involved in the heart failure telemonitoring service in Scotland. ${ }^{40}$ These included a general practitioner who had the initial responsibility for the telemonitoring program, three heart failure nurses who took over the monitoring from the general practitioner, and an information technology professional whose role was to support the service. The professional staff felt that telemonitoring enabled them to "see" their patients every day, not just once every few weeks or months, and that this enabled "... proactive approaches to clinical management ...". ${ }^{40}$ However, they also raised concerns that an increase in this form of communication may lead to increased patient dependence on the telemonitoring team as opposed to greater patient self-management (an issue discussed further on in this review), and for this reason considered that perhaps telemonitoring was best used for short periods of time to encourage medication adherence and until the patient's condition stabilized. Professionals were also concerned about the increased workload associated with telemonitoring as a result of checking data and increased communication with patients in response to the telemonitored data through follow-up phone calls and home visits. When queried about the impact on continuity of care and patient suitability for telemonitoring, the professional staff interviewed felt that it would be best to know the patient first, so the clinician could be aware of the patient's normal clinical parameters and that the telemonitored data were in context. For this reason perhaps, they thought that integration of telemonitoring with existing clinical relationships is preferable to centralized provision. ${ }^{40}$ 
The health professionals interviewed by Fairbrother et a ${ }^{40}$ raised some concern that patients may become overly dependent on monitoring by health professionals and self-care may decline ${ }^{40}$ In a qualitative study, Riley et al ${ }^{39}$ examined whether telemonitoring in heart failure empowers patients in terms of self-care, performing semistructured interviews with 15 patients (mean age 74 years, $73 \%$ male) ${ }^{39}$ at two time points, ie, after 3 and 6 months of telemonitoring. ${ }^{39}$ Telemonitoring was performed daily using a device that recorded and communicated weight, blood pressure, and pulse oximetry, as well as information on symptom. This study reported that patients " ... used telemonitoring to support their self-care actions ..." " 39 and developed greater understanding of heart failure through “... personal experience of symptoms, and their interaction with the telemonitoring and the telemonitoring nurse .....${ }^{39}$ In contrast with anecdotal evidence regarding some individuals being concerned that telemonitoring removes the personal interaction and relationship between the patient and health care professional, participants in this study felt that they developed a close relationship with the telemonitoring nurse and that rapport and trust were established. ${ }^{39}$ What is most interesting in the findings reported by Riley et al is that $33 \%$ of participants were aged over 80 years, highlighting that elderly patients are accepting and able to use telemonitoring equipment effectively and with a positive impact on their self-care. ${ }^{39}$

\section{What are the controversial issues? Types of technology}

As technology and health care advances, technologies that were once cutting edge become outdated. The current debate concerning the use of remote monitoring technology in the management of heart failure relates to the use of invasive monitoring devices (such as intrathoracic impedance monitoring) compared with external monitoring systems, such as noninvasive telemonitoring, structured telephone support (when the patient communicates data to a person, typically a nurse, following a standardized format), and interactive voice response systems. There were more studies of structured telephone support included in the Cochrane review; ${ }^{18}$ however, with the advent of new telemonitoring systems, better access to information communication technologies (such as Internet access, smart phone use, and network coverage) and the increasing focus on invasive monitoring, the evidence for noninvasive remote monitoring may be overtaken by invasive telemonitoring in the future. However, one technology that seems to be becoming less popular is interactive voice response systems, which are similar to those now being abandoned by many corporate customer service call centers, and not surprisingly, these appear to be less acceptable to patients. This was highlighted by the findings of the neutral Tele-HF study. ${ }^{33}$ After 6 months, only 55\% of participants were still using the system at least three times per week and $14 \%$ of participants randomized to the intervention never used the system. ${ }^{33} \mathrm{~A}$ recent post hoc analysis of findings from the large Cochrane review ${ }^{18}$ of telemonitoring and structured telephone support in heart failure that examined the impact of interactive voice response systems found no benefit on all-cause mortality or hospitalizations for chronic heart failure. ${ }^{42}$

\section{Patient preferences and patients unable to access face-to-face services}

Patient preference for delivery of care and ongoing monitoring should be taken into account, and is an important aspect of the use of telemonitoring. However, there is a significant mismatch in the supply and demand for heart failure management programs in many parts of the world. ${ }^{13,14}$ It would seem that the majority of patients with heart failure, especially those who live outside of major cities, do not have any option to access a heart failure management program, with only a minority of heart failure patients enrolled in a program. So where does that leave telemonitoring? If we cannot provide home or clinic visits to our heart failure patients who live outside major cities or where these services exist, then remote monitoring by way of telemonitoring or other forms may be a way to extend the reach of existing and future heart failure management programs.

There is also a feeling that patients may prefer to attend a clinic regularly or receive visits at home from health care providers for monitoring of symptoms, rather than interact via technology. Where patients with heart failure have access to a heart failure management program offering clinic or home visits, then these interventions may be most suitable for ongoing patient support and monitoring, and their benefits on patient outcomes are well accepted. ${ }^{43}$ As the evidence base for telemonitoring develops, this need not be at the cost of reducing face-to-face services where these are more appropriate, as long as we see telemonitoring and other forms of remote management as components in the management of heart failure which we can tailor according to the setting, population, and resources. Telemonitoring is a tool for remotely monitoring and supporting patients with heart failure, rather than a standalone intervention operating outside of the clinical supports and services otherwise available to patients. 


\section{Other controversial issues to consider}

Aside from access to information communication technology, there are several important aspects which need to be considered before initiating telemonitoring. These include the medicolegal and ethical aspects of collecting data; monitoring, altering, and initiating care based on the transferred data; and reimbursement and funding for telemonitoring, including costs of the equipment, setting up and maintenance of the equipment and resources, for monitoring and interpretation of the transferred data in order to care for the patient. ${ }^{44}$ Clinicians and health care providers want to know how often face-to-face assessments should be scheduled with patients whose heart failure is well managed when they are being telemonitored. There are also the considerations regarding the certification of health workers who manage and operate these systems and respond to the data. There is an increasing body of literature on these aspects of telemonitoring, and this falls outside the focus of this review.

\section{What does this mean for future research and clinical practice?}

What remains unclear in the current body of evidence regarding the use of telemonitoring in chronic heart failure is which parameters are best measured, in which patients, and how often and in what context in relation to other health care interventions and services? These are important aspects in the design and implementation of a telemonitoring program. How telemonitoring can influence, impact, or improve self-care is another aspect that warrants more examination, especially consideration of device designs and the monitoring protocols that best facilitate this.

It is no wonder that health care providers and planners may be hesitant to invest in these technologies when recent evidence demonstrates inconsistencies, many of which may be driven by not applying the most suitable combination of parameters, frequency, and response to the patients who are likely to benefit. In order to answer these questions we need to consider which patients will benefit from telemonitoring. It is unclear if this should be performed post-discharge for patients with a first or recurrent hospitalization for heart failure, especially those who cannot access other forms of regular specialized heart failure care and monitoring. Perhaps patients who cannot access standard heart failure programs due to mobility issues, or carer or employment commitments, would derive benefit? Many of the studies of telemonitoring in heart failure include mainly males ${ }^{18}$ and younger participants, so "real world" studies of telemonitoring in heart failure that include older patients and women will strengthen the evidence in these populations.

To avoid further confusion regarding studies of remote monitoring in heart failure, clear and consistent labeling of study interventions needs to be performed so that unnecessary heterogeneity is not introduced when comparing and interpreting the latest randomized controlled trial evidence for remote monitoring in heart failure. As a way to " ... enable accurate interpretation of clinical trials ...", Anker et al proposed a classification of remote monitoring according to " $\ldots$ the type of data transfer, the ... decision ability of the telemedical system, and the level of integration of all systems with the patient's primary care structure". ${ }^{12}$ Perhaps this system, along with clear labeling, will improve our understanding and interpretation of the evidence.

In terms of future clinical practice, there is great potential for telemonitoring to increase equity in accessing specialized heart failure monitoring and support for patients who, by virtue of geography, carer responsibilities, mobility issues, or other factors, are unable to regularly access specialized heart failure services. Additionally, an often overlooked aspect and benefit of telemonitoring and use of e-Health and m-Health interventions in heart failure care is the opportunity to provide culturally appropriate monitoring in heart failure through use of devices that are programmed in different languages and sensitive to different cultures (including dietary habits and patterns). Telemonitoring devices and programs may be best used as a way to offer and provide individualized and personalized approaches to remote monitoring and support.

\section{Conclusion}

It is important to note that telemonitoring alone does not improve patient outcomes; it is the monitoring, interpretation, and action regarding the data provided that may lead to improved outcomes for patients. Additionally, telemonitoring should be viewed as a component of multidisciplinary management of heart failure ${ }^{18}$ rather than as a standalone intervention. Most importantly, telemonitoring should be seen as a tool to assist patients and health care professionals to manage heart failure rather than as a reason to do away with the important role of nurses and other trained health care professionals in the ongoing management of heart failure. Since the publication of the Cochrane review, ${ }^{18}$ several studies of telemonitoring and remote interventions in heart failure have been published. ${ }^{29-33}$ An update of the Cochrane review, ${ }^{18}$ which is currently underway by the author of the present review, will seek to further delineate the facts 
regarding telemonitoring in heart failure, and based on the evidence, address some of the controversies in application of telemonitoring for heart failure.

\section{Acknowledgments}

The author is supported by a Cardiovascular Research Network Life Science Research Fellowship from the Heart Foundation and the NSW Office for Medical Research (CR 11S 6226).

\section{Disclosure}

The author is the lead author of the Cochrane Review cited in this work. No other conflicts of interest in this work.

\section{References}

1. Deeks JJ, Higgins JPT, Altman DG; on behalf of the Cochrane Statistical Methods Group. Analysing data and undertaking meta-analyses. In: Higgins JPT, Green S, editors. Cochrane Handbook for Systematic Reviews of Interventions. 2011. Available from: http://handbook. cochrane.org/. Accessed July 19, 2014.

2. Heidenreich PA, Albert NM, Allen LA, et al. Forecasting the impact of heart failure in the United States: a policy statement from the American Heart Association. Circ Heart Fail. 2013;6(3):606-619.

3. Pandya A, Gaziano TA, Weinstein MC, Cutler D. More Americans living longer with cardiovascular disease will increase costs while lowering quality of life. Health Aff (Millwood). 2013;32(10):1706-1714.

4. Davidson PM, Inglis SC, Newton PJ. Self-care in patients with chronic heart failure. Expert Rev Pharmacoecon Outcomes Res. 2013;13(3): 351-359.

5. Yancy CW, Jessup M, Bozkurt B, et al. 2013 ACCF/AHA guideline for the management of heart failure: a report of the American College of Cardiology Foundation/American Heart Association Task Force on Practice Guidelines. Circulation. 2013;128(16):e240-e327.

6. McMurray JJ, Adamopoulos S, Anker SD, et al. ESC Guidelines for the diagnosis and treatment of acute and chronic heart failure 2012: The Task Force for the Diagnosis and Treatment of Acute and Chronic Heart Failure 2012 of the European Society of Cardiology. Eur Heart J. 2012;33(14):1787-1847.

7. Konstam MA. Home monitoring should be the central element in an effective program of heart failure disease management/response to Konstam. Circulation. 2012;125(6):820-827.

8. de Lusignan S, Wells S, Johnson P, Meredith K, Leatham E. Compliance and effectiveness of 1 year's home telemonitoring. The report of a pilot study of patients with chronic heart failure. Eur J Heart Fail. 2001;3(6): 723-730.

9. Goldberg LR, Piette JD, Walsh MN, et al. Randomized trial of a daily electronic home monitoring system in patients with advanced heart failure: the Weight Monitoring in Heart Failure (WHARF). Am Heart J. 2003;146(4):705-712.

10. Florea V, Anand I. Clinical trial report: reevaluating telemonitoring in heart failure. Curr Heart Fail Rep. 2011;8(2):84-86.

11. Stoyanov N, Paul V. Clinical use of telemonitoring in chronic heart failure: keeping up with the times or misuse of time? Curr Heart Fail Rep. 2012;9(1):75-80.

12. Anker SD, Koehler F, Abraham WT. Telemedicine and remote management of patients with heart failure. Lancet. 2011;378(9792):731-739.

13. Clark RA, Driscoll A, Nottage J, et al. Inequitable provision of optimal services for patients with chronic heart failure: a national geo-mapping study. Med J Aust. 2007;186(4):169-173.

14. Clark RA, Driscoll A. Access and quality of heart failure management programs in Australia. Aust Crit Care. 2009;22(3):111-116.
15. Driscoll A, Worrall-Carter L, Hare DL, et al. Evidence-based chronic heart failure management programs: reality or myth? Qual Saf Health Care. 2009;18(6):450-455.

16. Driscoll A, Worrall-Carter L, McLennan S, Dawson A, O'Reilly J, Stewart S. Heterogeneity of heart failure management programs in Australia. Eur J Cardiovasc Nurs. 2006;5(1):75-82.

17. Jaarsma T, Strömberg A, De Geest S, et al. Heart failure management programmes in Europe. Eur J Cardiovasc Nurs. 2006;5(3):197-205.

18. Inglis SC, Clark RA, McAlister FA, et al. Structured telephone support or telemonitoring programs for patients with chronic heart failure. Cochrane Database Syst Rev. 2010;8:CD007228.

19. Klersy C, De Silvestri A, Gabutti G, Regoli F, Auricchio A. A metaanalysis of remote monitoring of heart failure patients. $\mathrm{J} \mathrm{Am} \mathrm{Coll}$ Cardiol. 2009;54(18):1683-1694.

20. Dang S, Dimmick S, Kelkar G. Evaluating the evidence base for the use of home telehealth remote monitoring in elderly with heart failure. Telemed J E Health. 2009; 15(8):783-796.

21. Clarke M, Shah A, Sharma U. Systematic review of studies on telemonitoring of patients with congestive heart failure: a meta-analysis. J Telemed Telecare. 2011;17(1):7-14.

22. Maric B, Kaan A, Ignaszewski A, Lear SA. A systematic review of telemonitoring technologies in heart failure. Eur J Heart Fail. 2009;11(5):506-517.

23. Polisena J, Tran K, Cimon K, et al. Home telemonitoring for congestive heart failure: a systematic review and meta-analysis. $J$ Telemed Telecare. 2010;16(2):68-76.

24. Xiang R, Li L, Liu SX. Meta-analysis and meta-regression of telehealth programmes for patients with chronic heart failure. JTelemed Telecare. 2013;19(5):249-259.

25. Pandor A, Gomersall T, Stevens JW, et al. Remote monitoring after recent hospital discharge in patients with heart failure: a systematic review and network meta-analysis. Heart. 2013;99(23):1717-1726.

26. Purcell R, McInnes S, Halcomb EJ. Telemonitoring can assist in managing cardiovascular disease in primary care: a systematic review of systematic reviews. BMC Fam Pract. 2014;15:43.

27. Conway A, Inglis SC, Chang AM, Horton-Breshears M, Cleland JG, Clark RA. Not all systematic reviews are systematic: a meta-review of the quality of systematic reviews for non-invasive remote monitoring in heart failure. J Telemed Telecare. 2013;19(6):326-337.

28. National Health and Medical Research Council. National Health and Medical Research Council Guide to the development, implementation and evaluation of clinical practice guidelines. Canberra, Australia. Available from: https://www.nhmrc.gov.au/_files_nhmrc/publications/ attachments/cp30.pdf. Accessed July 19, 2014.

29. Koehler F, Winkler S, Schieber M, et al. Impact of remote telemedical management on mortality and hospitalizations in ambulatory patients with chronic heart failure: the Telemedical Interventional Monitoring in Heart Failure Study. Circulation. 2011;123(17):1873-1880.

30. Lyngå P, Persson H, Hägg-Martinell A, et al. Weight monitoring in patients with severe heart failure (WISH). A randomized controlled trial. Eur J Heart Fail. 2012;14(4):438-444.

31. Boyne JJ, Vrijhoef HJ, Crijns HJ, De Weerd G, Kragten J, Gorgels AP; TEHAF investigators. Tailored telemonitoring in patients with heart failure: results of a multicentre randomized controlled trial. Eur J Heart Fail. 2012;14(7):791-801.

32. Dendale P, De Keulenaer G, Troisfontaines P, et al. Effect of a telemonitoring-facilitated collaboration between general practitioner and heart failure clinic on mortality and rehospitalization rates in severe heart failure: the TEMA-HF 1 (TElemonitoring in the MAnagement of Heart Failure) study. Eur J Heart Fail. 2012;14(3):333-340.

33. Chaudhry SI, Mattera JA, Curtis JP, et al. Telemonitoring in patients with heart failure. N Engl J Med. 2010;363(24):2301-2309.

34. Inglis SC, Clark RA, Cleland JG; Cochrane Systematic Review Team. Telemonitoring in patients with heart failure. $N$ Engl J Med. 2011;364(11):1078-1080.

35. Clark AM, Thompson DR. Telemedicine and remote management of heart failure. Lancet. 2011;378(9806):e9. 
36. Desai AS. Home monitoring heart failure care does not improve patient outcomes. Circulation. 2012;125(6):828-836.

37. Dar O, Riley J, Chapman C, et al. A randomized trial of home telemonitoring in a typical elderly heart failure population in North West London: results of the Home-HF study. Eur J Heart Fail. 2009;11(3): 319-325.

38. Inglis SC, Conway A, Cleland JG, Clark RA. Is age a factor in the success or failure of remote monitoring in heart failure? Telemonitoring and structured telephone support in elderly heart failure patients. Eur J Cardiovasc Nurs. March 29, 2015;14(3):248-255.

39. Riley JP, Gabe JPN, Cowie MR. Does telemonitoring in heart failure empower patients for self-care? A qualitative study. J Clin Nurs. 2013;22(17-18):2444-2455.

40. Fairbrother P, Ure J, Hanley J, et al. Telemonitoring for chronic heart failure: the views of patients and health care professionals - a qualitative study. J Clin Nurs. 2014;23(1-2):132-144.
41. Hall AK, Dodd V, Harris A, McArthur K, Dacso C, Colton LM. Heart failure patients' perceptions and use of technology to manage disease symptoms. Telemed J E Health. 2014;20(4):324-331.

42. Conway A, Inglis SC, Clark RA. Effective technologies for noninvasive remote monitoring in heart failure. Telemed $J$ E Health. 2014;20(6):531-538.

43. McAlister FA, Stewart S, Ferrua S, McMurray JJ. Multidisciplinary strategies for the management of heart failure patients at high risk for admission: a systematic review of randomized trials. JAm Coll Cardiol. 2004;44(4):810-819.

44. Lovell NH, Redmond SJ, Basilakis J, Shany T, Celler BG, editors. Telehealth technologies for managing chronic disease - experiences from Australia and the UK. Engineering in Medicine and Biology Society, 2010 Annual International Conference of the Institute of Electrical and Electronics Engineers, August 31 to September 4, 2010, Buenos Aires, Argentina.

\section{Publish your work in this journal}

Smart Homecare Technology and TeleHealth is an international, peer-reviewed, open access online journal publishing original research, reviews, editorials and commentaries on the application of technology to support people and patients at home and in assisted living centers to optimize healthcare and management resources. Specific topics in the journal include: Development and application of

\section{Dovepress}

devices within the home and embedded in appliances; Healthcare provider communication and education tools; and drug ordering and adherence. The manuscript management system is completely online and includes a very quick and fair peer-review system, which is all easy to use. Visit http://www.dovepress.com/ testimonials.php to read real quotes from published authors.

Submit your manuscript here: http://www.dovepress.com/smart-homecare-technology-and-telehealth-journal 